\title{
COMPARISON OF DRYING SHRINKAGE AND DRYING CREEP KINETICS IN CONCRETE
}

\author{
Lenka Dohnalová*, Petr Havlásek \\ Department of Mechanics, Faculty of Civil Engineering, Czech Technical University in Prague, Thákurova 7, \\ 16629 Prague 6, Czech Republic \\ * corresponding author: lenka.dohnalova@fsv.cvut.cz
}

\begin{abstract}
The aim of this paper is to show and compare the time evolution of drying shrinkage and drying creep in concrete from three different perspectives. The first one analyzes the basic equations defined in the most common design codes and prediction models for the description of the long-term behavior of concrete (ACI 209, EC2, Model Code 2010, B3, B4). Next, the evolution of drying creep and shrinkage is examined by processing suitable experimental data available from the database developed at the Northwestern University. Finally, the last point of view investigates the results obtained from the finite element simulations employing the material point approach, in particular, the material model based on the Microprestress-Solidification theory.
\end{abstract}

KEYworDS: Drying creep, shrinkage, concrete, design code, prediction model.

\section{INTRODUCTION}

Concrete drying is accompanied by its gradual contractive volume changes referred to as shrinkage and additional compliance called drying creep or the Pickett effect. On contrary to basic creep (i.e. creep of hygrally sealed specimens at room temperature), both drying shrinkage and drying creep are believed to be bounded.

The complex interplay between shrinkage and creep determines the evolution of stresses and so its thorough analysis can help to identify potential cracking and thus can contribute to estimation of the lifetime of the concrete structure. This is the case for members restrained either externally (statically indeterminate structures) or internally (nonuniform stress field generated by the steep gradient of the profile of relative humidity) but very often by the combination of both.

This paper summarizes and extends the final thesis of the first author [1].

The first part of this paper compares the time evolution of drying creep and drying shrinkage according to the most common design codes and prediction models. These models operate on the cross-sectional level and thus they provide the estimated average values of strain and compliance which are assumed to be uniform across the cross-section. Nowadays, most of the prediction models use as the input parameters the recipe of the concrete mixture, the 28-day compressive strength $\bar{f}_{c}$, and the duration of curing (or the onset of drying). In order to estimate the rate of drying (and so the rate of shrinkage and drying creep), it is necessary to provide the model with the size (usually by the quantity called "equivalent thickness" computed as the ratio of volume to drying surface) and the shape of the cross-section.

The second part of the paper examines and processes the available experimental data gathered in the database developed and maintained at the Northwestern University (NU) [2]. The next part compares the drying shrinkage and drying creep kinetics obtained from the results of the finite element simulations. These simulations operate on the material point level which implicates the non-uniform stress field. If such model is correctly calibrated, it is applicable not only for the analysis of complex and creep-sensitive structures, but also for the assessment of potential surface or internal cracking stemming from the severe drying conditions or excessive temperature generated by cement hydration in massive structures.

The results are compared and discussed in the last part of the paper.

\section{Design Codes and Prediction MODELS}

Two design codes (ACI 209.2R-08 [3] and Eurocode 2 [4]) and three prediction models for the long-term behavior of concrete (B3 model [5], B4 model [6] and fib Model Code 2010 [7]) were selected for a detailed analysis of time evolution of drying creep and drying shrinkage.

The investigated prediction models split concrete creep additively into basic creep and drying creep components allowing to evaluate drying creep directly. On the other hand, the analyzed design codes (ACI 209, Eurocode 2) provide only the formula for the total compliance; therefore, the drying creep compliance was obtained by subtracting the compliance at sealed conditions from the total compliance corresponding to the drying conditions.

The total shrinkage is usually considered as the sum of autogenous and drying shrinkage. The B3 model neglects the autogenous shrinkage, for this reason the drying shrinkage is set equal to the total shrinkage. 
The ACI 209 does not neglect the autogenous shrinkage but provides only the formula for total shrinkage which is nil at $100 \%$ relative humidity. Therefore, similarly to the B3 model, the total shrinkage is taken as the drying shrinkage.

Even though the individual models use different functions to define the time evolution of drying creep and shrinkage, the shape of the resulting curves is very similar. Bigger differences are observed in the final values but these are not in the scope of the present paper. Next, we present a brief description of the design and prediction models used in this study. Throughout the different models $\varepsilon_{s h, d}$ stands for the drying shrinkage and $h_{\mathrm{env}}$ for the ambient relative humidity (expressed as decimal). The expressions for the drying creep compliance $J_{d}$ are for simplicity presented with $t^{\prime}=t_{0}$, i.e. with the age at loading $t^{\prime}$ coinciding with the onset of drying $t_{0}$. The duration of loading/drying is denoted as $\hat{t}=t-t^{\prime}=t-t_{0}$. In some models the evolution of drying is derived from $V / S$ which is the ratio of the volume of the concrete member to its surface exposed to the ambient relative humidity.

B3 model was developed by Bažant and co-workers at the Northwestern University and uses, similarly to the other prediction models, empirical formulae to determine the time development of creep and shrinkage. The parameters in the model can be estimated based on the composition of the concrete mixture, the mean compressive strength and in the case of drying on the ambient relative humidity and on the size and shape of the concrete member. The autogenous shrinkage is neglected in the model and so the range of applicability is limited to the water-to-cement ratio above 0.35 .

The drying creep compliance and drying shrinkage are defined by the following two equations

$$
\begin{aligned}
& J_{d}(\hat{t})=q_{5}\left[e^{8\left(\left(1-h_{\mathrm{env}}\right) \cdot \tanh \sqrt{\frac{\hat{t}}{\tau_{s h}}}-1\right)}-1\right]^{0.5} \\
& \varepsilon_{s h}(\hat{t})=-\varepsilon_{s h}^{\infty} \cdot k_{h} \cdot \tanh \sqrt{\frac{\hat{t}}{\tau_{s h}}}
\end{aligned}
$$

Here, $\tau_{s h}$ is the shrinkage half-time depending on the size and shape of the member, $\varepsilon_{s h}^{\infty}$ is the absolute value of the ultimate drying shrinkage at $h_{\mathrm{env}}=0, k_{h}$ is a humidity-dependent factor, and $q_{5}$ is an adjustable parameter.

B4 model builds on the previous B3 model and uses more input parameters of the concrete mixture (e.g. aggregate type, additives and admixtures) as well as more elaborate formulae for the prediction of the model parameters. Nevertheless, the structure of basic equations of the B4 model is very similar to its predecessor. The autogenous shrinkage and drying shrinkage are treated in the B4 model separately. fib Model Code 2010 The next two equations present a simplified form of the drying creep compliance and shrinkage functions

$$
\begin{aligned}
& J_{d}\left(\hat{t}, t^{\prime}\right)=\frac{1}{E_{28}} \beta_{d c}^{*}\left[\frac{\hat{t}}{\beta_{D}+\hat{t}}\right]^{\frac{\sqrt{t^{\prime}}}{3.5+\sqrt{5.29 t^{\prime}}}} \\
& \varepsilon_{s h, d}(\hat{t})=\varepsilon_{s h, d 0} \beta_{R H}\left[\frac{\hat{t}}{0.035 D^{2}+\hat{t}}\right]^{0.5}
\end{aligned}
$$

where $E_{28}$ is the modulus of elasticity at the age of 28 days, $D$ is the effective cross section thickness (twice the volume-surface ratio), $\beta_{D}$ is a size dependence factor, $\beta_{R H}$ is a humidity dependence factor, and $\beta_{d c}^{*}$ and $\varepsilon_{s h, d 0}$ are model parameters depending on compressive strength, relative humidity and the age at loading.

Eurocode 2 does not specify an explicit formula for the evaluation of the compliance function $J$ from the creep coefficient $\varphi$, the conversion was done using a modified expression from Model Code 1990 [8].

$$
\begin{aligned}
& J\left(\hat{t}, t^{\prime}\right)=\frac{1}{E_{c m}\left(t^{\prime}\right)}+\frac{\varphi}{1.05 E_{c m}(28)}\left[\frac{\hat{t}}{\beta_{H}+\hat{t}}\right]^{0.3} \\
& \varepsilon_{s h, d}(\hat{t})=k_{D} \cdot \varepsilon_{s h 0} \cdot \frac{\hat{t}}{\hat{t}+0.04 \cdot D^{1.5}}
\end{aligned}
$$

where $E_{c m}\left(t^{\prime}\right)$ is the secant modulus of elasticity of concrete at the age of loading, $\beta_{H}$ is a parameter depending on relative humidity and member size, $k_{D} \cdot \varepsilon_{s h 0}$ expresses the ultimate value of drying shrinkage.

ACI 209.2R-08 On contrary to EC2, the American standard determines the compliance function from the creep coefficient and the secant modulus of elasticity at the age at loading $E_{c m}\left(t^{\prime}\right)$

$$
\begin{aligned}
& J\left(\hat{t}, t^{\prime}\right)=\frac{1}{E_{c m}\left(t^{\prime}\right)} \cdot\left[1+\frac{\hat{t}^{\psi}}{d+\hat{t}^{\psi}} \cdot \varphi_{u}\right] \\
& \varepsilon_{s h}(\hat{t})=\varepsilon_{s h u} \cdot \frac{\hat{t}^{\alpha}}{f+\hat{t}^{\alpha}}
\end{aligned}
$$

where $d, \psi, f$, and $\alpha$ are parameters, and $\varphi_{u}$ and $\varepsilon_{s h u}$ are the ultimate creep coefficient and the ultimate shrinkage, respectively, both influenced by the ambient relative humidity. There are two distinct options how to select the model parameters defining the time evolution of drying creep and shrinkage. The first one, as simplified in [3] from the former version of the code [9], is to use the average and fixed values of the parameters, then $\psi=0.6, \alpha=1.0$ and in general $d \neq f$. The second possibility, which reflects the size and shape of the structural member, is to set the exponents $\alpha=\psi=1.0$, and $d=f$ which are determined from $V / S$ of the drying member. 


\begin{tabular}{lcccccccccc}
\hline Basic parameters & $\begin{array}{c}\bar{f}_{c} \\
\mathrm{MPa}\end{array}$ & $\begin{array}{c}E_{c m 22} \\
\mathrm{GPa}\end{array}$ & $\begin{array}{c}t_{0} \\
\text { day }\end{array}$ & $\begin{array}{c}t^{\prime} \\
\text { day }\end{array}$ & $\begin{array}{c}c \\
\mathrm{~kg} \mathrm{~m}^{-3}\end{array}$ & $\begin{array}{c}w / c \\
-\end{array}$ & $\begin{array}{c}a / c \\
-\end{array}$ & $\begin{array}{c}\text { Cem. } \\
\text { type }\end{array}$ & $\begin{array}{c}h_{\mathrm{env}} \\
-\end{array}$ & $\begin{array}{c}2 V / S \\
\mathrm{~mm}\end{array}$ \\
\hline Example from ACI [3] & 33.3 & 28.2 & 7 & 7 & 409 & 0.50 & 4.23 & $\mathrm{I}$ & 0.7 & 200 \\
\hline Experiment Troxell [10] & 16.5 & $\mathrm{NA}$ & 28 & 28 & 320 & 0.59 & 5.67 & $\mathrm{I}$ & $0.5 / 0.7$ & 45 \\
\hline Experiment Bryant [1] & 50.1 & 29.8 & 8 & 8 & 390 & 0.47 & 5.09 & $\mathrm{I}$ & 0.6 & $150 / 75 *)$ \\
\hline Experiment Keeton [12] & 45 & 25.9 & 8 & 8 & 452 & 0.46 & 3.74 & $\mathrm{III}$ & $0.2 / 0.5$ & 65 \\
\hline
\end{tabular}

*) $150 \mathrm{~mm}$ slab, $75 \mathrm{~mm}$ prism

TABLE 1. Details of the experimental setup in the creep and shrinkage experiments.
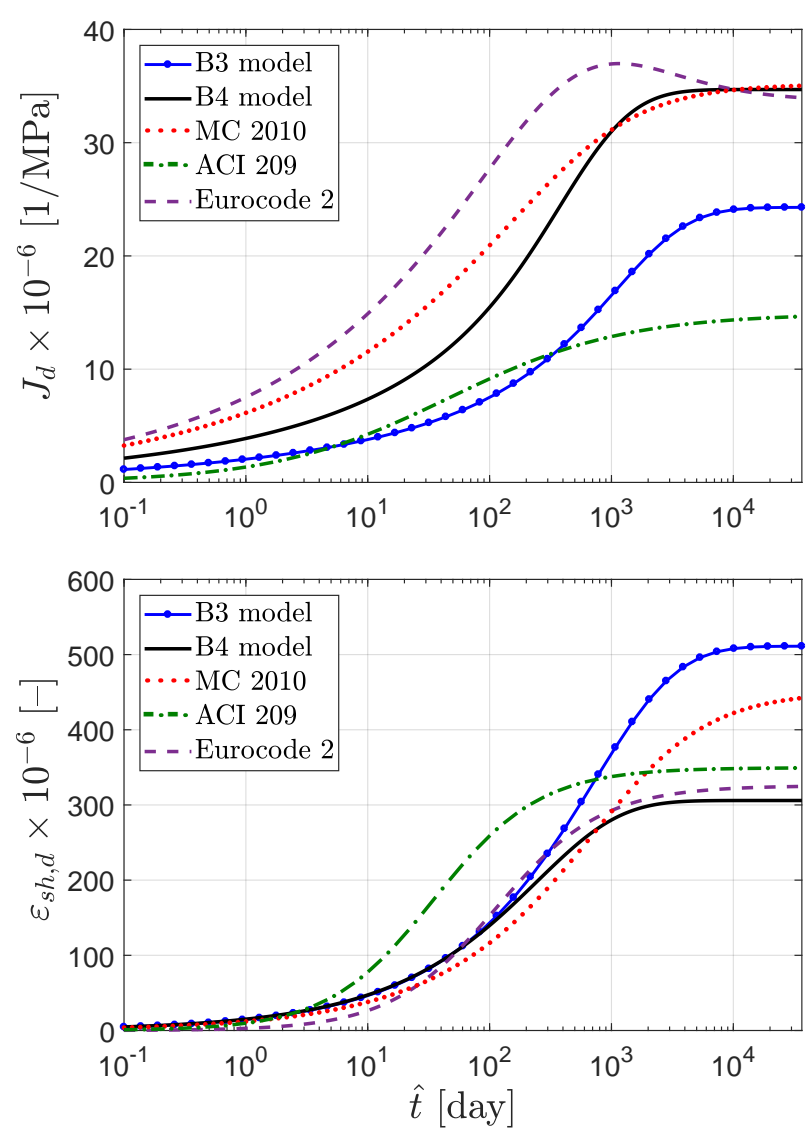

FIGURE 1. Drying creep compliance curves (top) and drying shrinkage curves (bottom) given by the analyzed design codes and prediction models.

The time evolution of drying creep and shrinkage shown in Fig. 1 was obtained with the parameters computed for the concrete mixture and the specimen properties defined in Appendix C of ACI 209 [3]. The parameters are listed in Tab. 1 The specimen is considered as a slab with $200 \mathrm{~mm}$ thickness and moist cured for 7 days. The aggregate type used in the B4 model is the quartzite. The curves in Fig. 1 are evaluated for the duration of 100 years which is the typical design period of most of the bridges and engineering structures. Both figures show the horizontal time axis in the logarithmic scale which is more suitable for processes with initially very high rate and which gradually decreases.

Fig. 2 presents the time evolution of normalized dry-
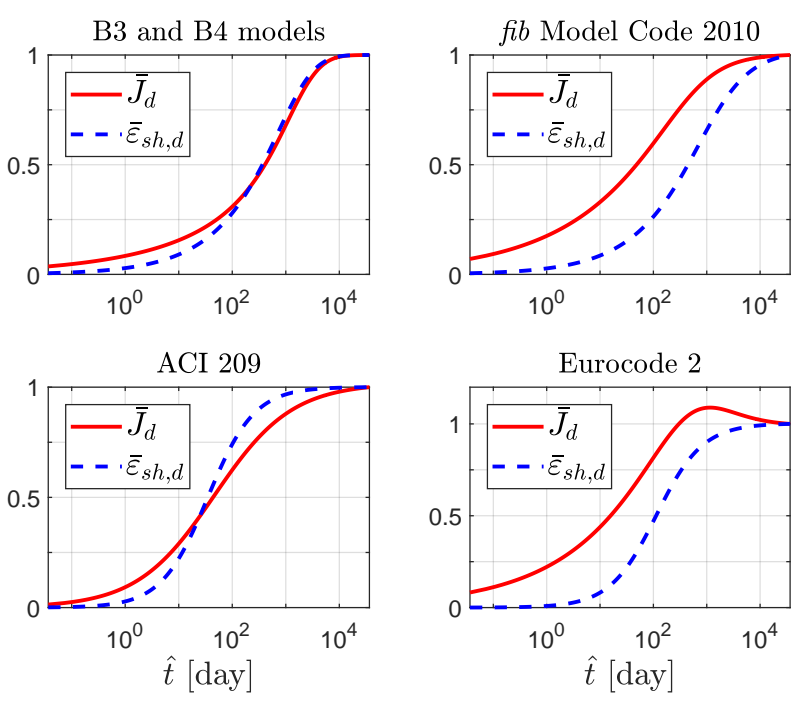

Figure 2. Time evolution of normalized drying creep and shrinkage according to the selected design codes and prediction models. The functions are normalized with respect to the ultimate value. Decreasing drying creep from EC2 model is physically unjustified and stems from the model formulation.

ing creep, $\left(\bar{J}_{d}\right.$, solid red lines) and drying shrinkage $\left(\bar{\varepsilon}_{s h, d}\right.$, dashed blue lines) for the selected design codes and prediction models. The evolution is normalized with respect to the ultimate value. The presented response of the models is based on the input parameters specified in the first line of Tab. 11. Naturally, the curves will be different for a modified composition, dimensions or other conditions, but the dominant trends will prevail provided that the ambient relative humidity is kept constant (see the first part in Section 5).

A different way of comparison is shown in Fig. 3 where the normalized drying creep is plotted against normalized drying shrinkage. However, here, the real kinetics of both phenomena is not reflected.

It must be noted that in the present example (shown in Figs. 1 3], the ACI model uses the recommended fixed values of parameters (i.e. the model is size independent). 


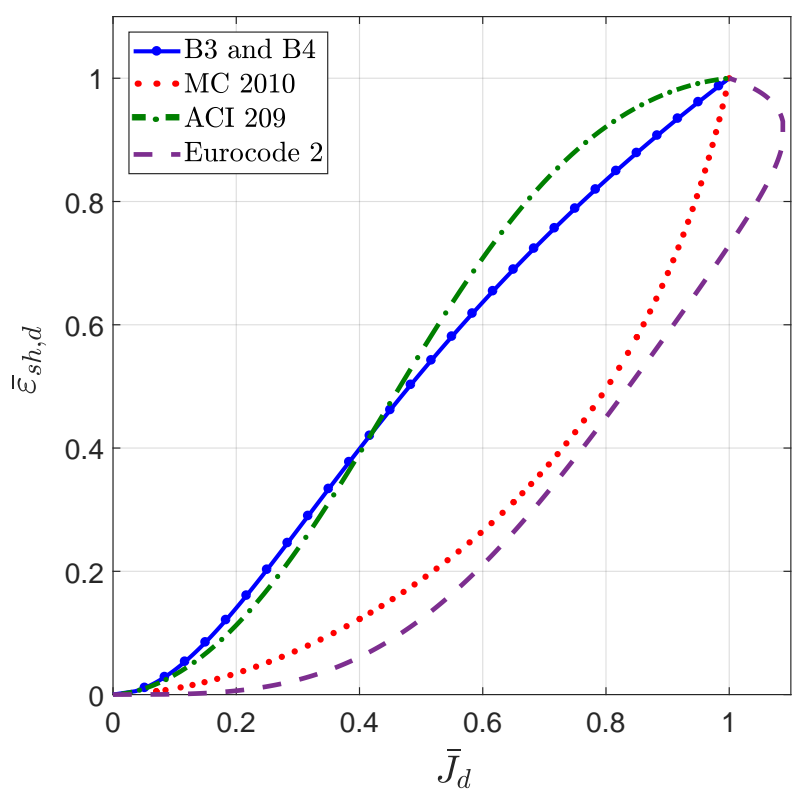

FIGURE 3. Comparison of normalized drying shrinkage and normalized drying creep evolution according to the selected design codes and prediction models. Normalization is done with respect to the ultimate value.

\section{EXPERIMENTAL DATA}

The freely available experimental database developed at the Northwestern University [2] was selected as the source of suitable experimental evidence of drying shrinkage and drying creep. Some experimental data comprised in this database were used for the calibration of the models presented in the previous section, and so it can be expected that the processed experimental data will very likely resemble the curves in Figs. 2 and 3.

The database contains 61,930 values measured on 3,308 specimens (1,439 for creep and 1,869 for shrinkage). These values come from 362 experimental surveys (172 creep, 190 shrinkage). Some experimental surveys analyzed more than one concrete composition; in total it was 378 in the case of creep and 652 different compositions in the case of shrinkage. Only 68 experimental surveys are relevant to this study because only those used the same recipe of the concrete mixture for both creep and shrinkage specimens.

For the present purpose it was necessary to further narrow the selection only to those experiments which tested simultaneously drying shrinkage on unloaded specimens, basic creep, and total creep matter. Additionally, the loading in the creep experiments had to be applied simultaneously with the beginning of drying of both loaded and unloaded specimens.

The database contains only very few surveys which provide the information on autogenous shrinkage and sometimes it is difficult to distinguish between experiments carried out at sealed conditions and experiments where the specimens are immersed in water or exposed to very high relative humidity and thus exhibit swelling. In order to eliminate this deficiency an additional criteria were set on the value of water-to-cement ratio (minimum value $w / c=0.4$ ) and the compressive strength (maximum value $\bar{f}_{c}=60 \mathrm{MPa}$ ), then the autogenous shrinkage can be treated as negligible compared to the magnitude of the drying shrinkage.

Only 97 sets from 9 experimental surveys met the aforementioned criteria. Unfortunately, it was necessary to further reduce the selection because of incorrect or missing pieces of information, or too short duration of the experiment. The duration was judged as sufficient if the rate of drying creep and shrinkage was minimal at the end of the experiment. This was satisfied only in 6 experimental surveys (with 71 specimens) out of which 3 representative are partially presented in this paper.

The value of drying creep was determined as the difference between total and basic creep; when needed (in the case of different times of measurement), the value was computed using linear interpolation between two adjacent measurements. The drying creep and shrinkage was afterwards normalized with respect to the last value attained.

The suitable experiments are summarized in the following paragraphs.

Experiments of Troxell [10] adopted in this study are part of an extensive experimental survey focused on the influence of different aspects on the development of creep and shrinkage. The experiment started in 1928 and its duration exceeded 20 years. The data used in this study come from the experimental series \#3 which examined the influence of relative humidity; the specimens were cylinders with $102 \mathrm{~mm}$ diameter and $356 \mathrm{~mm}$ height. The basic properties and the composition of the concrete mixture are specified in Tab. 1

The NU database does not contain data for $\hat{t}<12$ days and thus completely misses the initial evolution of creep and shrinkage. Two triplets of specimens were suitable for the comparison of drying shrinkage and drying creep kinetics, one for $50 \%$, the second one for $70 \%$ relative humidity. The results are shown in Fig. 4 .

Experiments of Bryant [1] was initially focused on study of creep and shrinkage in bridge structures. Only specimens with dimensions $150 \times 150 \times 600 \mathrm{~mm}$ were suitable for the present study because only this size was loaded at the age when the drying began which was not the case of the remaining sizes. The specimens should have realistically represented structural members - slabs and pillars, to achieve this, one set of specimens had both ends and two opposite lateral sides hygrally sealed (specimens referred to as "slab") which resulted in unidirectional drying. The other set was sealed only at the ends and thus the specimens were drying evenly from all lateral sides, this set is denoted as "prism". 


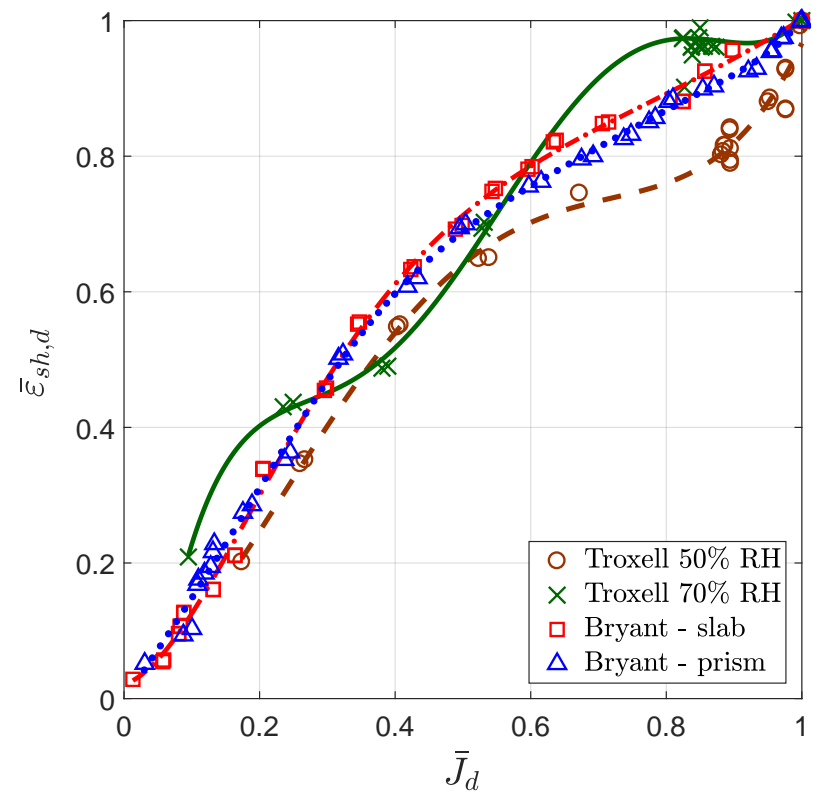

FIGURE 4. Comparison of normalized drying shrinkage and normalized drying creep evaluated from the experiments of Troxell and Bryant. Only the points represent the experimental data.

At the age of 8 days all specimens were transferred to the environment with $60 \%$ relative humidity. Unfortunately, during the experiment the aluminum foil used for sealing has corroded and especially the entirely sealed specimens started unintentionally drying resulting into an increase in creep or shrinkage rate. For this reason the autogenous shrinkage was neglected and the total shrinkage was taken as the drying shrinkage. At the later stage of the experiment, the measurements of the basic creep (spoiled by the sealing leak) partially influenced the determination of drying creep. The processed results are for both groups (slabs and prisms) shown in Fig. 4.

Experiments of Keeton 12] The suitable experimental data come from the part of research studying the influence of ambient relative humidity, specimen size, and the load level on creep and shrinkage. The research started in 1957 and lasted for 897 days. The data are not from the NU database but instead from the original paper (and are different). In several cases the increments of basic creep are larger than the increments of total creep. This was the problem especially of smaller specimens with lower level of loading and obviously specimens exposed to higher of relative humidity. Additionally, both loaded and unloaded companion specimens were not sealed but were subjected to $100 \%$ relative humidity. Owing to the aforementioned reasons the smaller specimens, the specimens with low loading level and specimens drying at $75 \%$ relative humidity are omitted from the present analysis.

Fig. 5 shows processed results obtained from experiments on cylinders $152 \times 457 \mathrm{~mm}$ drying at $20 \%$ and $50 \%$ relative humidity and loaded at $30 \%$ and $40 \%$ of

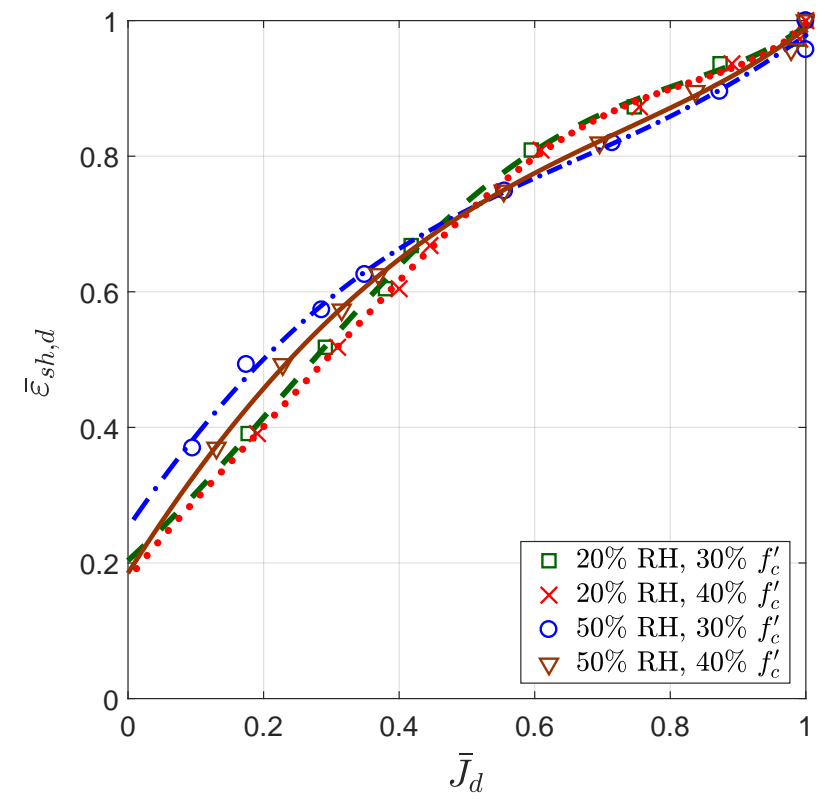

FiguRE 5. Comparison of normalized drying shrinkage and normalized drying creep evaluated from the experiments of Keeton at $20 \%$ and $50 \%$ relative humidity and two levels of loading. $f_{c}^{\prime}$ is the compressive strength at the age of loading. Points are the experimental data.

the compressive strength at the age of loading, $f_{c}^{\prime}$. In this figure, the higher initial level of shrinkage stems from the first measurement in which the total creep was smaller than the basic creep.

\section{Data From Finite Elements Simulations}

Except for the prismatic specimens $150 \times 150 \times 600 \mathrm{~mm}$ shown in the previous section of this study, the Bryant's experimental survey comprises the information on shrinkage and drying creep measured on geometrically specimens ranging from $100 \times 100 \times 400 \mathrm{~mm}$ to $400 \times 400 \times 1600 \mathrm{~mm}$ prepared from a single concrete batch. For this reason this experiment was in [13] and [14] selected as an ideal opportunity for verification of the material model based on MicroprestressSolidification theory (MPS) [15].

It turned out that the original MPS gives the opposite size effect on drying creep than the experiment and for this reason the MPS model was modified [13, 14. Here we present the comparison of drying creep and shrinkage for both approaches. Hereafter, the modified version of MPS model is referred to as "MPS improved". The presented data use the processed results obtained from the finite element simulations carried out in the open source FE package [16. The problem was analyzed as one-way coupled with the structural problem depending on the moisture transport according to the Bažand and Najjar model [17] for diffusion of water vapor. In order to obtain the shrinkage and drying creep kinetics, it was necessary perform, similarly to the real experiment, 3 analy- 

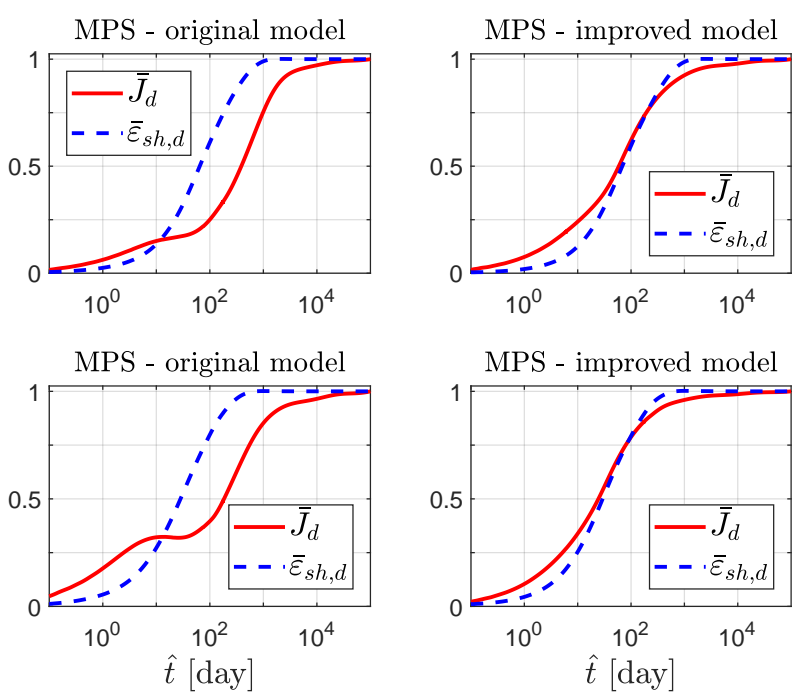

Figure 6 . The processed results of FE simulations of Bryant's experiment showing the time evolution of normalized drying creep and shrinkage. The top two figures are for $150 \mathrm{~mm}$ slabs bottom two figures are for $150 \times 150 \mathrm{~mm}$ prism. The curves are normalized with respect to the ultimate value.

ses: basic creep, drying creep and shrinkage. The values of the material parameters in the models were selected in order to obtain a reasonably accurate fit of all specimens ("prisms" and "slabs" of different sizes), therefore, the agreement of the simulation of $150 \mathrm{~mm}$ specimen with the experimental data cannot be expected to be satisfactory.

The normalized drying creep and shrinkage is for the original and improved MPS model and the two geometries shown in Fig. 6

\section{Discussion}

In general, according to the prediction models and design codes, the time evolution of drying creep and drying shrinkage depends dominantly on the ambient relative humidity and on the size of the concrete member, the influence of other factors is not so significant.

When evaluating the differences in kinetics of drying creep and drying shrinkage, each model is sensitive only to certain input parameters.

In the case of the $\mathbf{B} \mathbf{3}$ model, the only factor which affects the relationship between drying creep and drying shrinkage is the ambient relative humidity. At low relative humidity, the drying creep is delayed behind shrinkage, but on contrary to this, at high levels $\left(h_{\text {env }}>0.8\right)$, the delay is in shrinkage. However, the difference is in both cases relatively small, see Fig. 7

On the other hand, the fib MC2010 is completely insensitive to $h_{\mathrm{env}}$. The kinetics is influenced dominantly by the size of the member (its $V / S$ ), see Fig. 8 . The larger the size the more is the curve in Fig. 2 bent downwards and the bigger is the delay of shrinkage behind drying creep. This is caused by the fact that with decreasing $V / S$ the drying shrinkage becomes

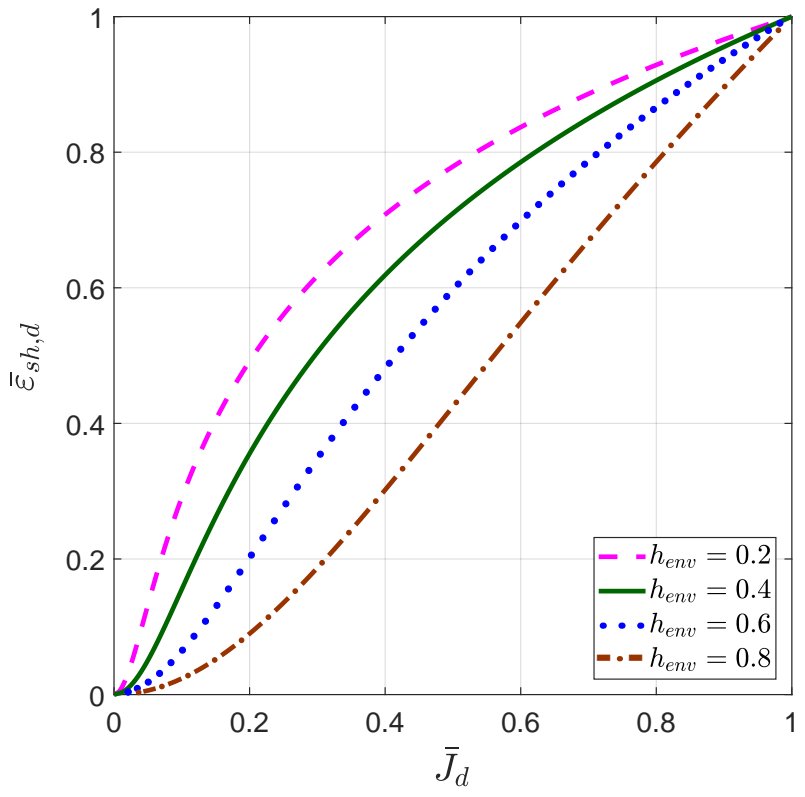

Figure 7 . The influence of different levels of ambient relative humidity on the drying creep vs. drying shrinkage kinetics according to the B3 model. The remaining parameters are from the first line in Tab. 1

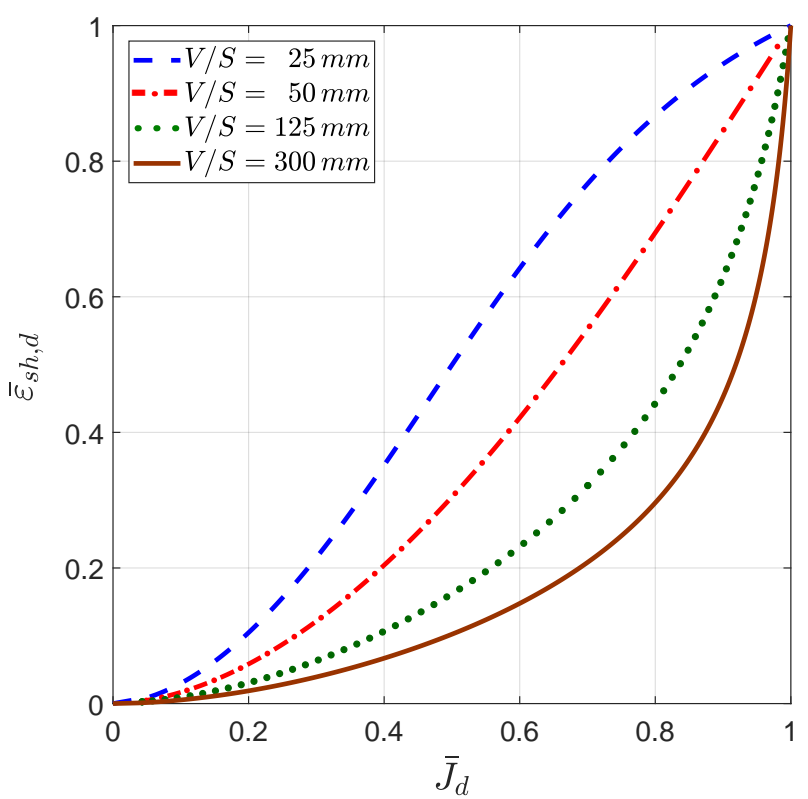

Figure 8. The influence of different $V / S$ ratios on the drying creep vs. drying shrinkage kinetics according to the fib MC 2010 model. The remaining parameters are from the first line in Tab. 1

more accelerated than the drying creep. To certain extent the kinetics is also influenced by the onset of drying, but for reasonable values the differences are not substantial.

The Eurocode 2 predicts, except for extreme combinations of parameters, delay in shrinkage behind drying creep. The model is sensitive both to $V / S$ a and $h_{\text {env }}$. For smaller specimens and higher relative humidity the drying creep curve becomes more non-monotonic. 


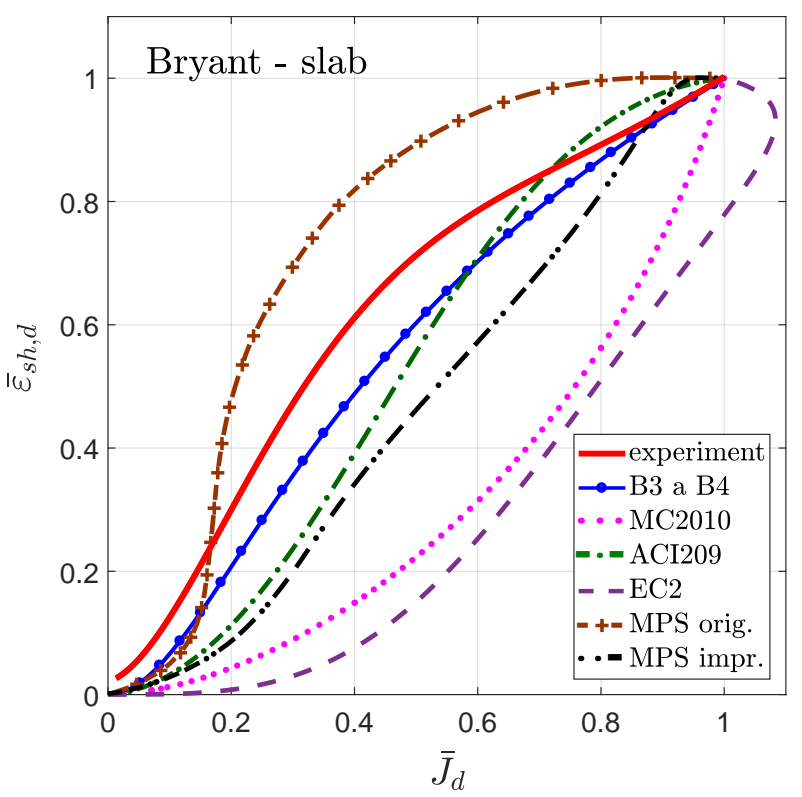

Figure 9. The comparison of the experimental data, prediction models and FE simulations of the Bryant's experiment, $2 \mathrm{~V} / \mathrm{S}=150 \mathrm{~mm}$ (slab).

ACI 209 is the exception to the other presented models. For first approach with the average recommended values (according to [9]) the kinetics of drying creep and shrinkage remains constant, with an initial small delay of shrinkage behind drying creep. According to the second approach, which sets equal the exponents $\alpha$ and $\psi$ as well as the parameters $d$ and $f$, the kinetics of drying creep and drying shrinkage become identical (i.e. the function would be a diagonal in Fig. 3).

As presented in Figs. 4 and 5, the selected suitable experimental data give the opposite trend compared the prediction models (except for B3/4 at lower relative humidity and ACI at the later stage of drying). This means that in the experiments the drying shrinkage is a little bit ahead of the drying creep. The points in these figures correspond to the experimental data which are interpolated by the curves of the same color.

Two issues have to be pointed out. First, the resulting shape of the curve in the normalized plot shrinkage vs. drying creep depends on the duration of the experiment. Insufficiently long experiments can lead to misleading conclusions. Second, as demonstrated in Fig. 5, the readings should be documented more frequently in the initial phase of drying and the specimens should be instrumented as soon as possible. In the case of Keeton's experiment, the first displayed data point corresponds to the drying duration 7 days and the shrinkage strain reaches almost $40 \%$ of the last measured value (after approx. 3 years). (The first data points for $\hat{t}=1$ day which would have yielded negative $J_{d}$ is omitted here.)

According to the computations with the original version of the material model based on the MicroprestressSolidification theory, the drying creep evolves in an

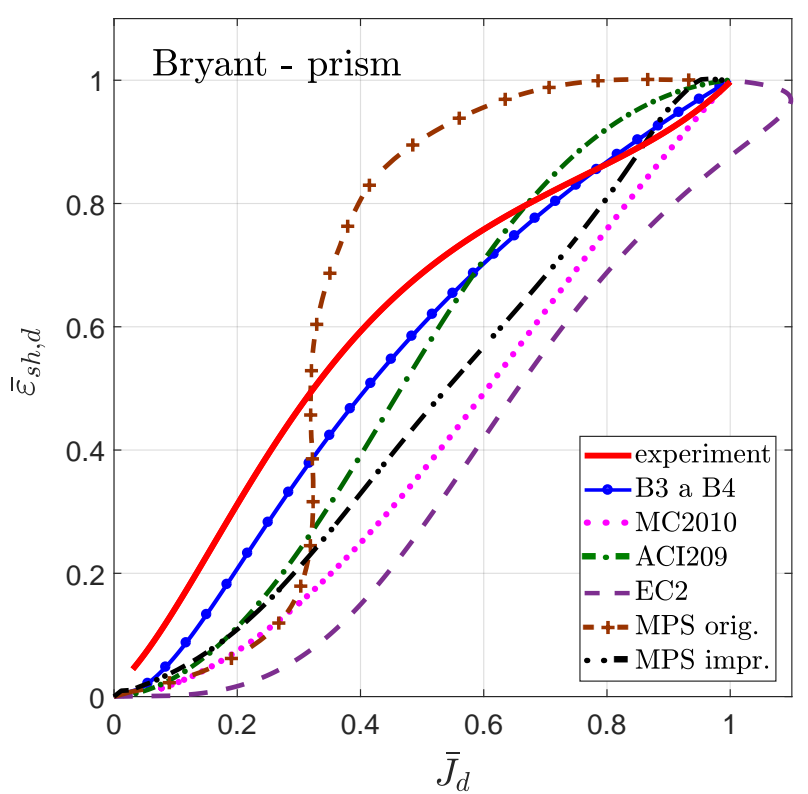

Figure 10. The comparison of the experimental data, prediction models and FE simulations of the Bryant's experiment, $2 \mathrm{~V} / \mathrm{S}=75 \mathrm{~mm}$ (prism).

unexpected fashion. First, as shown in the two left graphs in Fig. 6 and in Figs. 9 and 10, the drying creep evolves initially faster than drying shrinkage, then, suddenly, the creep stops and after certain time it continues with a considerable delay after shrinkage. This effect is more pronounced in the case of prismatic shape which dries more rapidly.

The response of the improved MPS model is more promising, see the two right graphs in Fig 6. Similarly to the cross-sectional approaches, it gives a small initial delay of shrinkage after drying creep, afterwards, the relationship is almost linear. However, the initial delay is reversed compared to the analyzed experimental data.

Very probably, in the case of the models working on the cross-sectional approach, the differences of the shrinkage and drying creep kinetics origin from their optimization and calibration procedure. Very likely the models were calibrated against the entire database with evenly distributed weights and without emphasizing that some specimens are companions belonging to the same experimental survey.

\section{Conclusions}

The presented study analyzed the time evolution of drying shrinkage $\varepsilon_{s h, d}$ and drying creep $J_{d}$ from three different perspectives: the most common design and prediction models, experimental data measured on laboratory concrete specimens, and the finite element simulations.

The following conclusions can be drawn:

- Even though the design codes and prediction models are calibrated on a very similar experimental database, $J_{d}$ and $\varepsilon_{s h, d}$ are described in each model 
differently and their kinetics is different. Additionally, in each model, the relationship between the $J_{d}$ and $\varepsilon_{s h, d}$ depends on different factors. Three of the analyzed models, B3, B4, and ACI 209, give for the standard conditions comparable kinetics of these processes. However, in the case of fib MC2010 and EC2 the delay of $\varepsilon_{s h, d}$ behind $J_{d}$ is substantial. According to $\mathrm{EC} 2, J_{d}$ is a non-monotonic function.

- A closer inspection of the experimental database which contains a vast amount of data revealed that only very few experiments fulfill the needs of the present research. Unfortunately, most of the experiments does not last sufficiently long or certain essential pieces of information are missing. The analyzed data indicate that the $J_{d}$ is initially slightly delayed behind $\varepsilon_{s h, d}$, which means that the trend is exactly opposite compared to the prediction models.

- The improved MPS model gives more realistic kinetics of $J_{d}$ and $\varepsilon_{s h, d}$ compared to its predecessor which unexpectedly gives a significant decrease in drying creep rate in the middle of drying. Both models predict, similarly to the cross-sectional models, an initial small delay of $\varepsilon_{s h, d}$ behind $J_{d}$.

- Sufficiently long experiments on normal-strength concrete are vital for the development and proper calibration of the material models for creep and concrete, however, they are very scarce.

\section{ACKNOWLEDGEMENTS}

The authors gratefully acknowledge financial support from the Grant Agency of the Czech Technical University in Prague, project number SGS18/037/OHK1/1T/11. The numerical analyses have been performed with OOFEM, an open-source object-oriented finite element program [16, 18.

\section{REFERENCES}

[1] L. Dohnalová. Comparison of shrinkage and drying creep kinetics of concrete, Bc. Thesis. Czech Technical University in Prague, 2018.

[2] M. Hubler, R. Wendner, Z. Bažant. Comprehensive database for concrete creep and shrinkage: analysis and recommendations for testing and recording. ACI 112:547-558, 2015.

[3] ACI Committee 209. Guide for Modeling and Calculating Shrinkage and Creep in Hardened Concrete. ACI, 2008 .

[4] Český normalizační institut. Eurokód 2: Navrhování betonových konstrukcí - Část 1-1: Obecná pravidla a pravidla pro pozemní stavby. Český normalizační institut, 1996.
[5] Z. Bažant, S. Baweja. Creep and shrinkage prediction model for analysis and design of concrete structures: Model B3. Adam Neville Symposium: Creep and Shrinkage - Structural Design Effects 2000.

[6] RILEM Technical Committee TC-242-MDC (Z.P. Bažant, chair). Model B4 for creep, drying shrinkage and autogenous shrinkage of normal and high-strength concretes with multi-decade applicability (RILEM Technical Committee TC-242-MDC multi-decade creep and shrinkage of concrete: material model and structural analysis). Materials and Structures 48:753-770, 2015.

[7] Fédération Internationale du Béton. Model Code 2010. No. vol. 65 in fib Bulletin. International Federation for Structural Concrete (fib), 2012.

[8] Comité Euro-International du Béton (CEB). CEB-FIP Model Code 1990: Design Code. T. Telford, London, 1993.

[9] A. C. 209. Prediction of Creep, Shrinkage, and Temperature Effects in Concrete Structures. American Concrete Institute, 1997.

[10] G. Troxel, J. Raphael, R. Davis. Long-time creep and shrinkage tests of plain and reinforced concrete. Proc ASTM 58 pp. 1101-1120, 1958.

[11] A. H. Bryant, C. Vadhanavikkit. Creep, shrinkage-size, and age at loading effects. ACI Materials Journal 84:117-123, 1987.

[12] Keeton. Study of Creep in Concrete, Phase 1 (I-beam). U. S. Naval Civil Engineering Laboratory, Port Hueneme, California, 1965.

[13] P. Havlásek. Creep and Shrinkage of Concrete Subjected to Variable Environmental Conditions, PhD. Thesis. Czech Technical University in Prague, 2014.

[14] Z. Bažant, P. Havlásek, M. Jirásek. Microprestress-solidification theory: Modeling of size effect on drying creep. In N. Bicanic, H. Mang, G. Meschke, R. de Borst (eds.), Computational Modelling of Concrete Structures, pp. 749-758. CRC Press/Balkema, EH Leiden, The Netherlands, 2014.

[15] Z. P. Bažant, A. P. Hauggaard, S. Baweja, F. J. Ulm. Microprestress solidification theory for concrete creep. I: Aging and drying effects. Journal of Engineering Mechanics 123:1188-1194, 1997.

[16] B. Patzák. OOFEM home page, 2000. Http://www.oofem.org.

[17] Z. P. Bažant, L. J. Najjar. Nonlinear water diffusion in nonsaturated concrete. Materials and Structures 5:3-20, 1972. DOI:10.1007/BF02479073

[18] B. Patzák. OOFEM - an object-oriented simulation tool for advanced modeling of materials and structures. Acta Polytechnica 52(6):59-66, 2012. 